\title{
Effects of different dark chocolate diets on memory functions and brain corticosterone levels in rats under chronic stress
}

\author{
Elham Kalantarzadeh, Maryam Radahmadi* iD, Parham Reisi \\ Department of Physiology, School of Medicine, Isfahan University of Medical Sciences, Isfahan, Iran
}

\begin{abstract}
Introduction: Stress influences cognitive behavior adversely, whereas dark chocolate exhibits positive effects on memory processes. This study investigated the effects of different dark chocolate diets on various aspects of brain functions in rats under chronic stress
\end{abstract}

Methods: Thirty-five rats were randomly allocated into five groups: control, stress, stress with different (compulsory, optional and restricted) dark chocolate diets. Latency, dark stay (DS) time and the number of entrance to the dark compartment were respectively evaluated as memory, memory consolidation and locomotor activity by passive avoidance test.

Results: There were significant differences between initial latency and latency after 1 day in all groups. In the stress-compulsory and restricted dark chocolate diet groups, latency after 1 day increased significantly. Moreover, the DS time was not significantly higher in the stressed group than the control group. The DS time and number of entrance to dark compartment decreased significantly in the stress-compulsory dark chocolate diet group compared to the stressed group. Furthermore, the number of entrance to dark compartment was significantly higher for the stress- optional dark chocolate diet compared to those with the compulsory diet. Additionally, serum and hippocampal corticosterone levels, except in the frontal cortex, were significantly lower only in the stress-compulsory dark chocolate diet group compared to the stressed group.

Conclusion: Different dark chocolate diets had various effects on brain functions under chronic stress. Respectively, the compulsory and optional dark chocolate diets had the best and least effects on brain function improvement. Only the compulsory dark chocolate diet could improve brain functions such as memory, memory consolidation and locomotor activity.

http://dx.doi.org/10.32598/ppj.24.3.40

\section{Keywords:}

Dark chocolat;

Memory;

Stress;

Hippocampus;

Frontal cortex

* Corresponding author:

M. Radahmadi

Email:

m_radahmadi@med.mui.ac.ir

Tel: +98 (031) 37929176

Received 5 January 2020;

Received in revised form 22

April 2020; Accepted 4 May

2020

\section{Introduction}

Stress is the state of psychological response to actual or potential stressors and activates the hypothalamic- pituitary-adrenal axis and glucocorticoids (the stress hormones) secretion (Dedovic et al., 2009; Radahmadi et al., 2015). Also, stress impairs some 
brain functions such as learning, memory, memory consolidation and behavioral activities by glucocorticoid secretion (Dastgerdi et al., 2018; Miranda and Oliveira, 2015). Hippocampus and frontal cortex are structures that have glucocorticoid receptors (Allan et al., 2014; Barrientos et al., 2015) and are known to be responsible for memory, moods, cognition and stress-responsive neuroendocrine systems (Cerqueira et al., 2005; Kim and Han, 2016; Radahmadi et al., 2014; Sugimoto et al., 2017). Hence, the functional changes in both hippocampus and frontal cortex under stressful conditions could impair brain activities (Holtzer et al., 2017; Li et al., 2011; Radahmadi et al., 2014).

In recent years, herbal drugs, their products and bioactive substances are considered to be alternative remedial strategies for some disorders due to their easy access and lower side effects (Bandegi et al., 2014). As such, chocolate, derived from cocoa beans, is a worldwide product that has been promoted as a functional food during the past decade (Franco et al., 2013). Besides, there is an increasing in the potential benefits of cocoa and chocolate for health (Steinberg et al., 2003). Cocoa and kinds of chocolate have antioxidants and phytochemicals like flavonoids that participate in improving deficits in brain function (Crichton et al., 2016). Cocoa flavonoids can exhibit beneficial impacts on cognition, mood and behavior (Sokolov et al., 2013). There were different kinds of chocolate including white, milk and dark chocolates, based on the amount of cocoa (Crichton et al., 2016; Kemsawasd et al., 2016); however, among the various kinds, dark chocolate is most effective type on brain functions and glucocorticoid levels (Martin et al., 2009; Sokolov et al., 2013). Correspondingly, the consumption of dark chocolate may optimize cognitive functions over the life span of an individual (Crichton et al., 2016), especially under stress conditions (Simadibrata, 2011). Furthermore, dietary changes are major lifestyle factors that can influence the progression of chronic disorders (Shah et al., 2017). Even though. the positive effects of dark chocolate consumption in reducing stress responses have been discussed before (Martin et al., 2009), none of the former studies have indicated how dark chocolate consumption may affect different aspects of brain function under stress. Therefore, this study aims to investigate the impacts of three different dark chocolate dietary patterns on some of the brain functions, such as learning, memory, memory consolidation and locomotor activity in rats under chronic stress, so the dark chocolate diet for the protection of brain corticosterone levels in serum, hippocampus, and frontal cortex under stress conditions could be introduced.

\section{Materials and methods}

\section{Experimental animals}

Thirty-five male Wistar rats (initial weight 250-300g) were obtained from Pasteur Institute of Tehran, Iran. The rats were housed under controlled humidity $(50 \pm 5 \%)$ and light conditions (12h light/dark; lights on 07:00-19:00), in the cages with similar size among all groups. The room temperature was set to $23 \pm 2^{\circ} \mathrm{C}$ and water was made available ad libitum. All behavioral experiments were performed at 13:0014:00 and the experiment lasted for a period of 14 days. The Ethics Committee of Animal Use at the Isfahan University of Medical Sciences approved the study (IR.MUI.MED.REC.1398.036) and all experiments were conducted in compliance with the National Institute of Health Guide for the Care and Use of Laboratory Animals (NIH Publications No. 8023, revised 2011). After a period of 2 weeks for adaptation, the animals were randomly divided equally into five groups $(n=7)$ : 1 - control group, the rats were maintained in the cage with no special treatment for 14 days and only received rodent's food (chow); 2- stress (St) group, the rats were subjected to isolation stress. Each rat was placed inside a cage alone and only received chow for 14 days; 3 - stress with compulsory dark chocolate diet (St-Compulsory Choc) group, the rats were subjected to isolation stress. Each rat was placed inside a cage alone and only received dark chocolate for 14 days; 4- stress with optional dark chocolate diet (St-Optional Choc) group, the rats were subjected to isolation stress. Each rat was placed inside a cage alone and freely received dark chocolate and chow for 14 days. Therefore, rats could optionally eat dark chocolate and/or chow; 5- stress with restricted dark chocolate diet (St-Restricted Choc) group, the rats were subjected to isolation stress. Each rat was placed inside a cage alone and freely received chow and only $4 \mathrm{~g} / \mathrm{rat} /$ day of dark chocolate for 14 days. Hence, rats could limitedly eat dark chocolate. 


\section{Experimental procedures Stress paradigm}

In this study to induce isolation stress in rats, they were kept inside separate cages without any neighbors (individual housing) for 14 days (Iglesias et al., 1992; Mills and Ward, 1984).

\section{Chocolate consumption}

Each rat consumed the specified amount of solid $85 \%$ dark chocolate $(588 \mathrm{kcal} / 100 \mathrm{~g}, 70.8 \%$ fat, $21.6 \%$ protein, $10.98 \%$ carbohydrate and other minerals; Shirin-Asal Co., Iran). The stressed rats in the compulsory and optional dark chocolate diets were respectively fed with only dark chocolate in the compulsory diet, and chow/chocolate in the optional diet while placed in the separate cages. Also, the stressed rats with a restricted dark chocolate diet were fed with chow and only the specified amount of dark chocolate $(4 \mathrm{~g} / \mathrm{rat} /$ day $)$ in their separate cage, calculated based on the human equivalent dose (Shin et al., 2010). However, another study has reported a respective chocolate consumption of $5 \mathrm{~g} / \mathrm{rat} / \mathrm{day}$ (Dailey et al., 2012).

\section{Behavioral Paradigms}

To assess learning, memory, memory consolidation and locomotor activity involving the cognitive memory, the passive avoidance (PA) test was used (Dastgerdi et al., 2018; Vohora et al., 2000). The PA apparatus (shuttle-box $64 \times 25 \times 35 \mathrm{~cm}$ ) was divided into two identical compartments $(32 \times 25 \times 35 \mathrm{~cm})$ with sliding guillotine doors and grid floors. On day 12 , each rat was placed in the apparatus for 300 s for habituation. On the following day (Day 13), a single learning trial was performed and on day 14, the passive avoidance memory trial was conducted. There were no foot shocks in both habituation and memory trials. In the learning trial, the rats were individually placed in the light compartment for 60s before the guillotine door was raised. When the rat entered the dark compartment, the door was closed and a single electric shock (0.5mA, $50 \mathrm{v}$ and $2 \mathrm{~s}$; once) was delivered to the animal's foot through the grid floor using an isolated stimulator (Huang et al., 2013). The initial latency time to enter the dark compartment was recorded before inducing the electric shock. In the memory trial, the latency time of entry to the dark compartment was measured after one day (up to a maximum delay of $300 \mathrm{~s}$ ). If the rat did not enter the dark compartment within 300 s, the memory trial was terminated. In this experiment, the difference between the initial latency and the latency after 1 day was translated as the occurrence of learning (Dastgerdi et al., 2018). Moreover, the total dark stay (DS) time was assigned as either memory consolidation and/or storage of new information (Dastgerdi et al., 2018). Similarly, the number of entrance into the dark compartment was recorded as the locomotor activity (Divsalar, 2012; Vohora et al., 2000). The animal's ability to remember the received foot-shock was determined by the PA test. Avoidance to enter the dark compartment or even a longer duration of stay in the light compartment was interpreted as a positive response (Dastgerdi et al., 2018).

\section{Assessment of corticosterone levels in the serum, hippocampus and frontal cortex}

On day 15, the rats were anesthetized with Urethane $(1.5 \mathrm{~g} / \mathrm{kg}$ IP, Sigma-Aldrich Chemical Co.) and sacrificed between 14:00-16:00. Subsequently, the serum was separated from blood samples and the hemi-hippocampus and hemi-frontal cortex were immediately dissected to be kept on dry ice. Furthermore, they were separately immersed in Problock $^{\mathrm{TM}}$-50, EDTA free (GoldBio Co., USA) and in phosphate buffer solution (0.01M, pH 7.4) (Dastgerdi et al., 2017). The hippocampus and frontal cortex were homogenized and centrifuged at $10,000 \mathrm{~g}$ for $20 \mathrm{~min}$ at $4^{\circ} \mathrm{C}$. The supernatant fluids were collected, subsequently both serum and supernatants were stored at $-80^{\circ} \mathrm{C}$ until the assessment time. The commercial Enzyme-Linked Immuno-Sorbent Assay kit (ZellBio GmbH Co., Marburg, Germany) was used to assess the serum, hippocampal and frontal cortical corticosterone levels.

\section{Statistical analysis}

All passive avoidance test data, corticosterone levels in serum, hippocampus and frontal cortex were analyzed by analysis of variance (ANOVA) test, followed by LSD post-hoc testing for multiple groups. The comparisons between the initial latency and latency after 1 day (within groups) were analyzed using the paired sample t-test. Furthermore, all data were estimated as mean \pm SEM. A $P$-value less than 0.05 was considered statistically significant. Notably, the calculations were performed using 


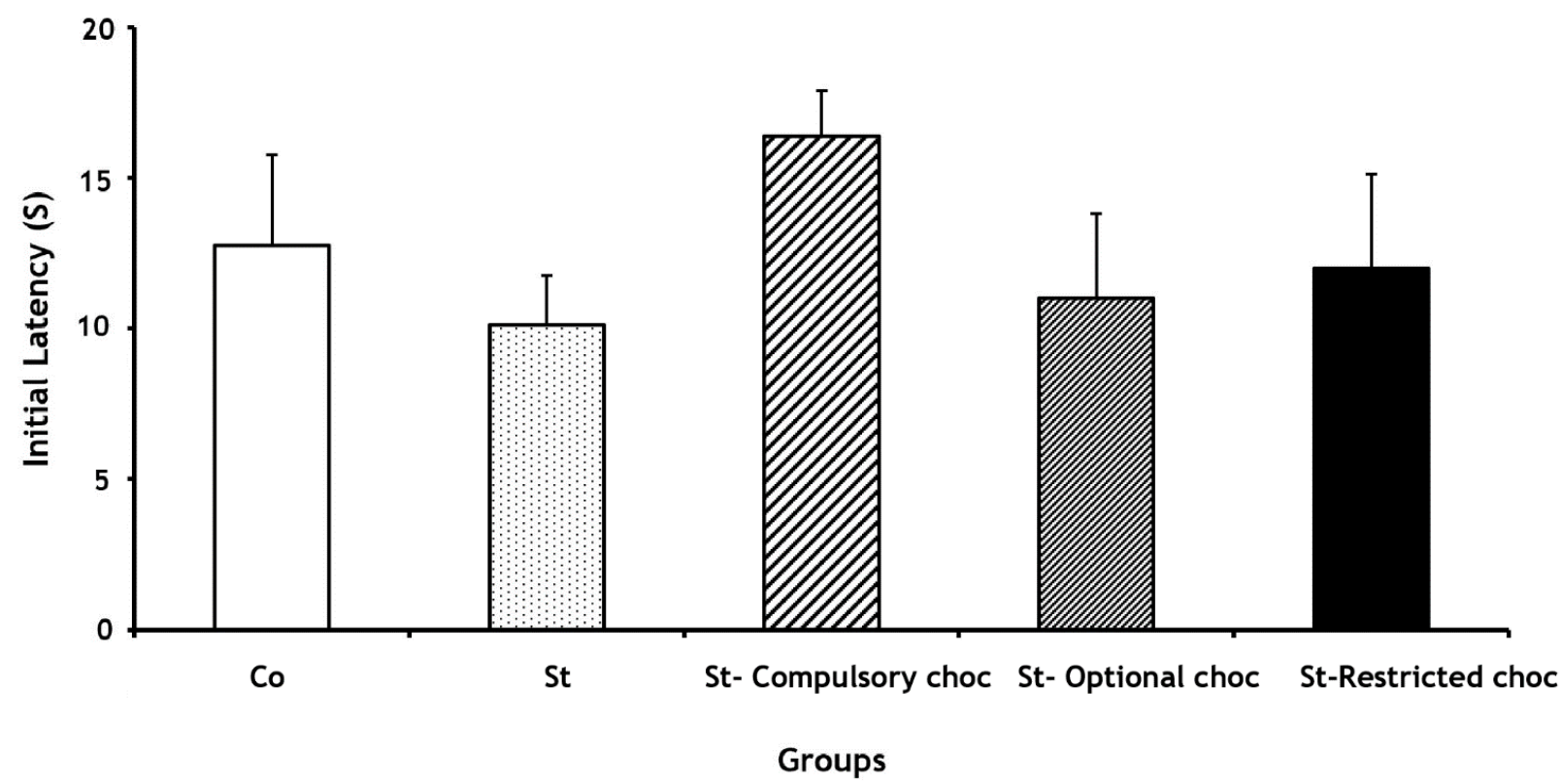

Fig.1. Initial latency to entrance into the dark room of the passive avoidance apparatus for all the groups before receiving a foot shock $(n=7)$. Results are expressed as mean \pm SEM. No significant differences were observed among the groups. Co: control group, St: stress group, St- Compulsory Choc: stress with compulsory dark chocolate diet group, St-Optional Choc: stress with optional dark chocolate diet group, St-Restricted Choc: stress with restricted dark chocolate diet group.

SPSS v21.0 (SPSS Inc., Chicago, IL, USA).

\section{Results}

In the passive avoidance test, ANOVA indicated no significant differences of the initial latency $[F(4,35)=-$ $0.915, P>0.05$; Fig. 1], total dark stay time $[F(4,35)=-$ 1.847, $P>0.05$; Fig. 4], and the number of entrance into the dark compartment $[F(4,35)=1.833, P>0.05$; Fig. 5]. Whereas, there was a significant latency difference after 1 day $[F(4,35)=2.661, P<0.05$; Fig. 2].

\section{Assessment of memory functions}

Figures 1 and 2, respectively, show the initial latency and latency after 1 day for all groups. No significant differences were observed in initial latency values across all groups (Fig. 1). In the stress (St) group, the latency after 1 day was significantly $(P<0.05)$ lower than that value recorded for the control group, indicating the memory declination as a result of chronic isolation stress (Fig. 2). The latency showed significant enhancements in St-Compulsory Choc and St-Restricted Choc groups (respectively, $P<0.01$ and $P<0.05)$ in comparison with the $S t$ group, representing the improving effects of compulsory and even restricted dark chocolate diets on memory deficit caused by stress after 1 day. However, the optional dark chocolate diet had no major impact on memory improvement in stressed rats (Fig. 2).
As shown in Figure 3, the initial latency and latency after 1 day were analyzed using a paired sample ttest to evaluate within-group latency changes. There were significant differences between initial latency and latency, determined by the PA test in the control $[\mathrm{t}(7)=$ $-7.169, \quad P>0.01]$, St $[t(7)=-3.859, \quad P>0.01]$, StCompulsory Choc $[\mathrm{t}(7)=-4.387, P>0.01]$, St-Optional Choc $[\mathrm{t}(7)=-2.787, P>0.05]$ and St-Restricted Choc $[t(7)=-4.303, P>0.01]$ groups (Fig. 3). Therefore, significant differences were detected between the initial latency and latency after 1 day in all groups. It indicated that the occurrence of learning in all groups, nevertheless at different levels. For instance, the learning happened at the lowest level in the St group and the highest level in the St-Compulsory Choc group.

The total DS time in the St group was not significantly higher than what was recorded for the Co group. However, the total DS time in the St-Compulsory Choc group was significantly $(P<0.05)$ lower than the DS in the St group, indicating that compulsory dark chocolate diet improved the memory consolidation. Whereas, the restricted and optional dark chocolate diet groups were not significantly different compared to the control and St groups (Fig. 4).

The number of entrance into the dark compartment showed no significant differences in St group compared to that recorded for the control group. Also, 


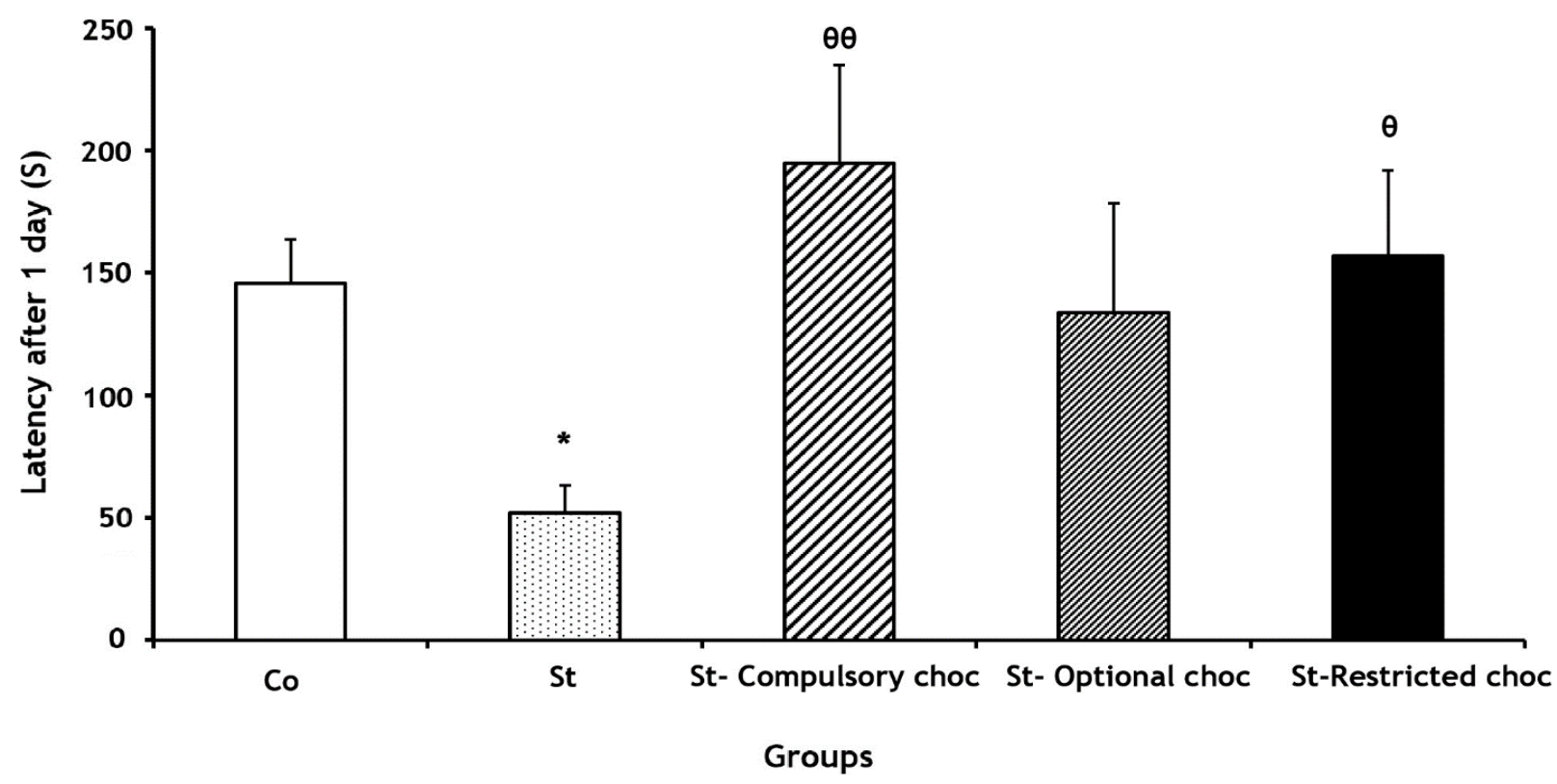

Fig.2. Latency after 1 day to entrance into the dark room of the passive avoidance apparatus for all the groups 1 day after receiving the foot shock $(n=7)$. Results are expressed as mean \pm SEM. ${ }^{*} P<0.05$ compared to control group; ${ }^{\ominus} P<0.05$ and ${ }^{\ominus \ominus} P<0.01$ compared to stress group. Co: control group, St: stress group, St- Compulsory Choc: stress with compulsory dark chocolate diet group, St-Optional Choc: stress with optional dark chocolate diet group, St-Restricted Choc: stress with restricted dark chocolate diet group.

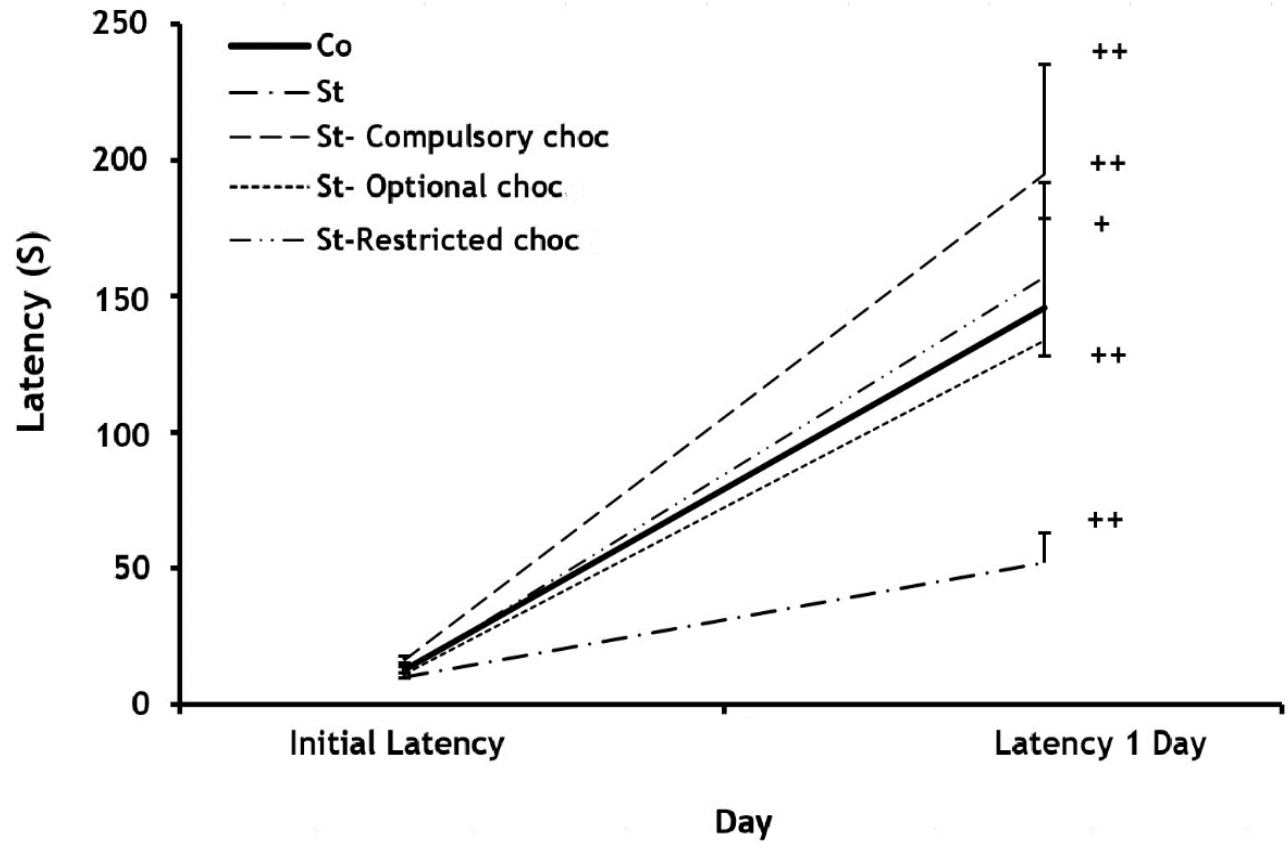

Fig.3. Initial latency and latency after 1 day to entrance into the dark room of the passive avoidance apparatus before and after the foot shock (within groups) $(\mathrm{n}=7)$. Results are expressed as mean \pm SEM. ${ }^{+} P<0.05$ and ${ }^{++} P<0.01$ Initial latency relative to the latency after 1 day. Co: control group, St: stress group, St- Compulsory Choc: stress with compulsory dark chocolate diet group, St-Optional Choc: stress with optional dark chocolate diet group, St-Restricted Choc: stress with restricted dark chocolate diet group.

in the St-Compulsory Choc group, it was significantly $(P<0.05)$ lower than the St group, indicating a reduced locomotor activity due to a compulsory dark chocolate diet under stress conditions. As such, the number of entries to the dark compartment were not significantly different in the restricted and optional dark chocolate diet groups compared to the St group (Fig. 5). In addition, the number of entrance into dark 


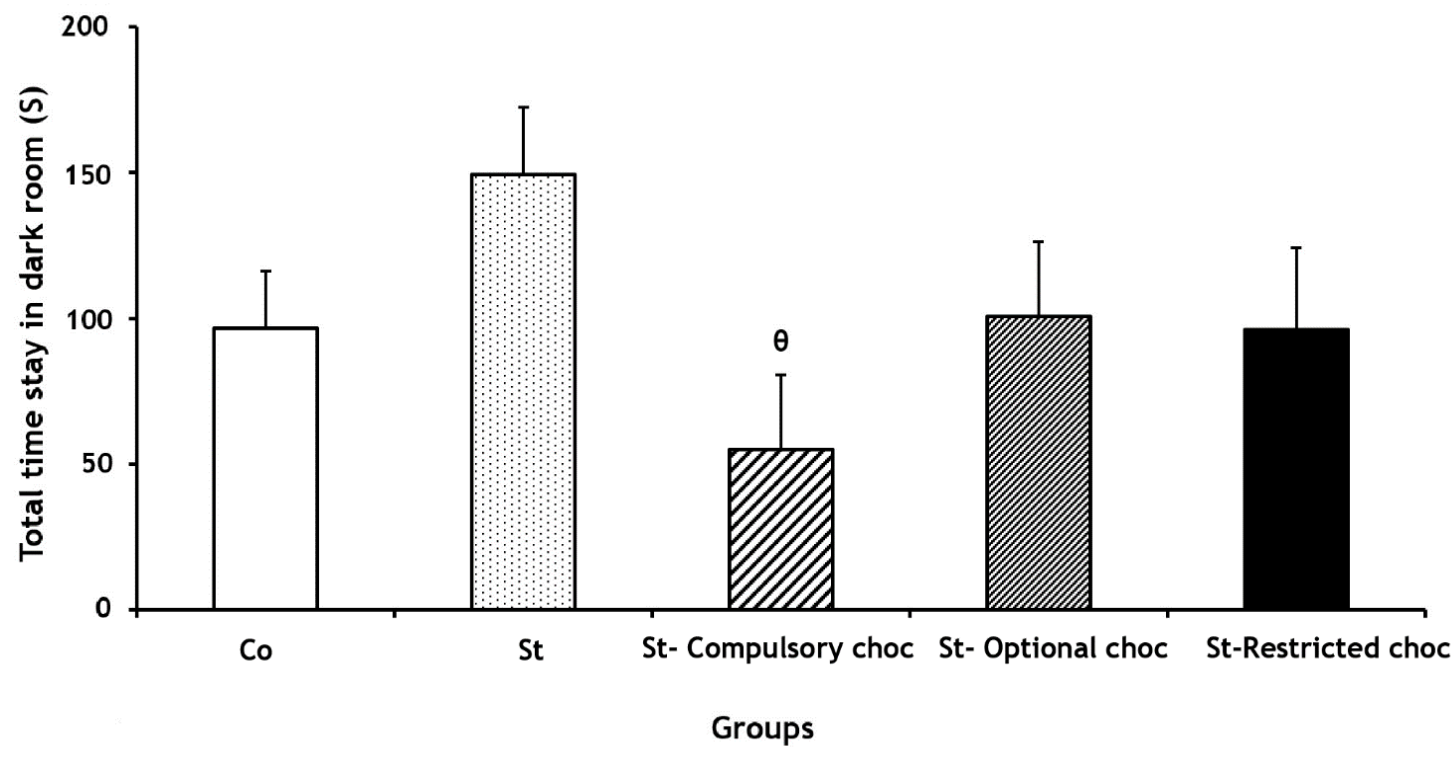

Fig.4. Total stay time in dark room of the passive avoidance apparatus for all the groups 1 day after receiving the foot shock $(\mathrm{n}=7)$. Results are expressed as mean \pm SEM. ${ }^{\ominus} P<0.05$ compared to stress group. Co: control group, St: stress group, St- Compulsory Choc: stress with compulsory dark chocolate diet group, St-Optional Choc: stress with optional dark chocolate diet group, St-Restricted Choc: stress with restricted dark chocolate diet group.

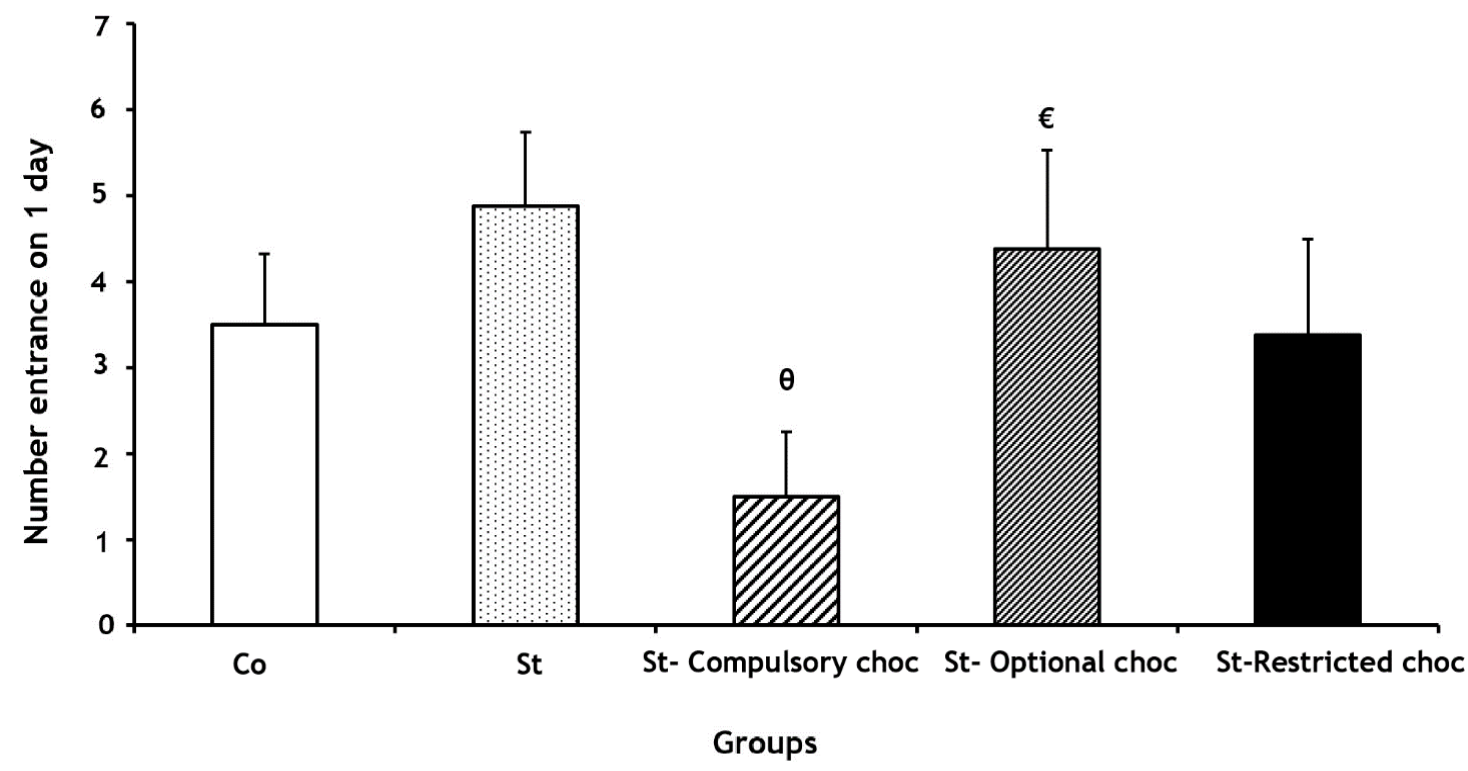

Fig.5. The number entrance to dark room of the passive avoidance apparatus for all the groups 1 day after receiving the foot shock $(\mathrm{n}=7)$. Results are expressed as mean \pm SEM. ${ }^{\ominus} P<0.05$ compared to stress group; ${ }^{€} P<0.05$ compared to stresscompulsory choco group. Co: control group, St: stress group, St- Compulsory Choc: stress with compulsory dark chocolate diet group, St-Optional Choc: stress with optional dark chocolate diet group, St-Restricted Choc: stress with restricted dark chocolate diet group.

compartment in the St-Optional Choc diet group increased significantly $(P<0.05)$ in comparison with the St-Compulsory dark chocolate diet group, displaying an elevated locomotor activity in this group (Fig. 5).

Assessment of corticosterone levels in the

\section{serum, hippocampus and frontal cortex}

In the assessment of corticosterone levels, the ANOVA test indicated a significant difference in serum corticosterone levels $[F(4,35)=4.309, P>0.01$; Fig. 6]. Whereas, there were no significant differences in hippocampal corticosterone levels $[F(4$, $35)=1.587, P>0.05$; Fig. 7] and in the frontal cortical 


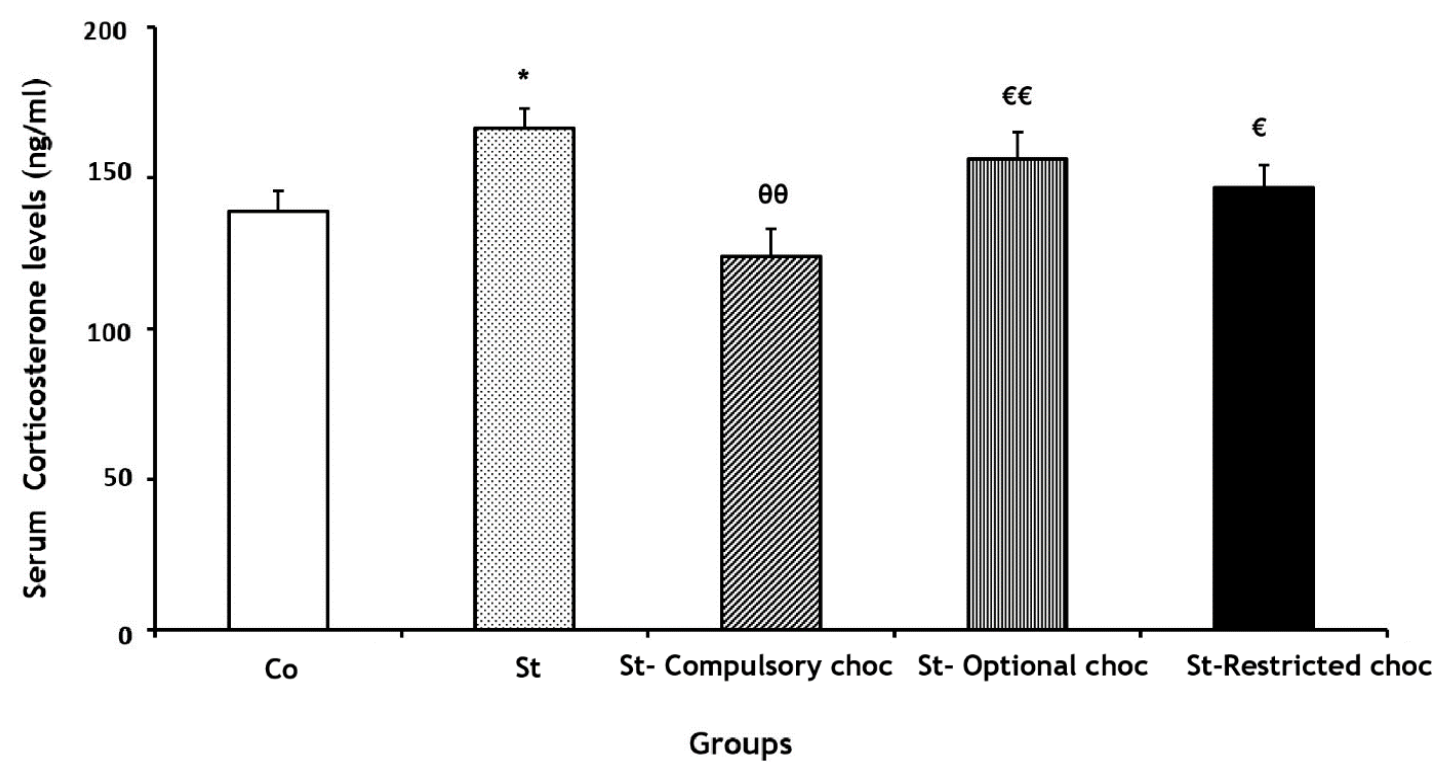

Fig.6. Effects of different dark chocolate diets on serum corticosterone levels $(\mathrm{ng} / \mathrm{ml})$ in different groups under isolation stress $(\mathrm{n}=7)$. Results are expressed as mean \pm SEM. ${ }^{*} P<0.05$ compared to control group, ${ }^{\ominus \ominus} P<0.01$ compared to stress group, ${ }^{€} P<0.05$ and ${ }^{€}{ }^{€}<0.01$ compared to stress- compulsory choco group. Co: control group, St: stress group, StCompulsory Choc: stress with compulsory dark chocolate diet group, St-Optional Choc: stress with optional dark chocolate diet group, St-Restricted Choc: stress with restricted dark chocolate diet group.

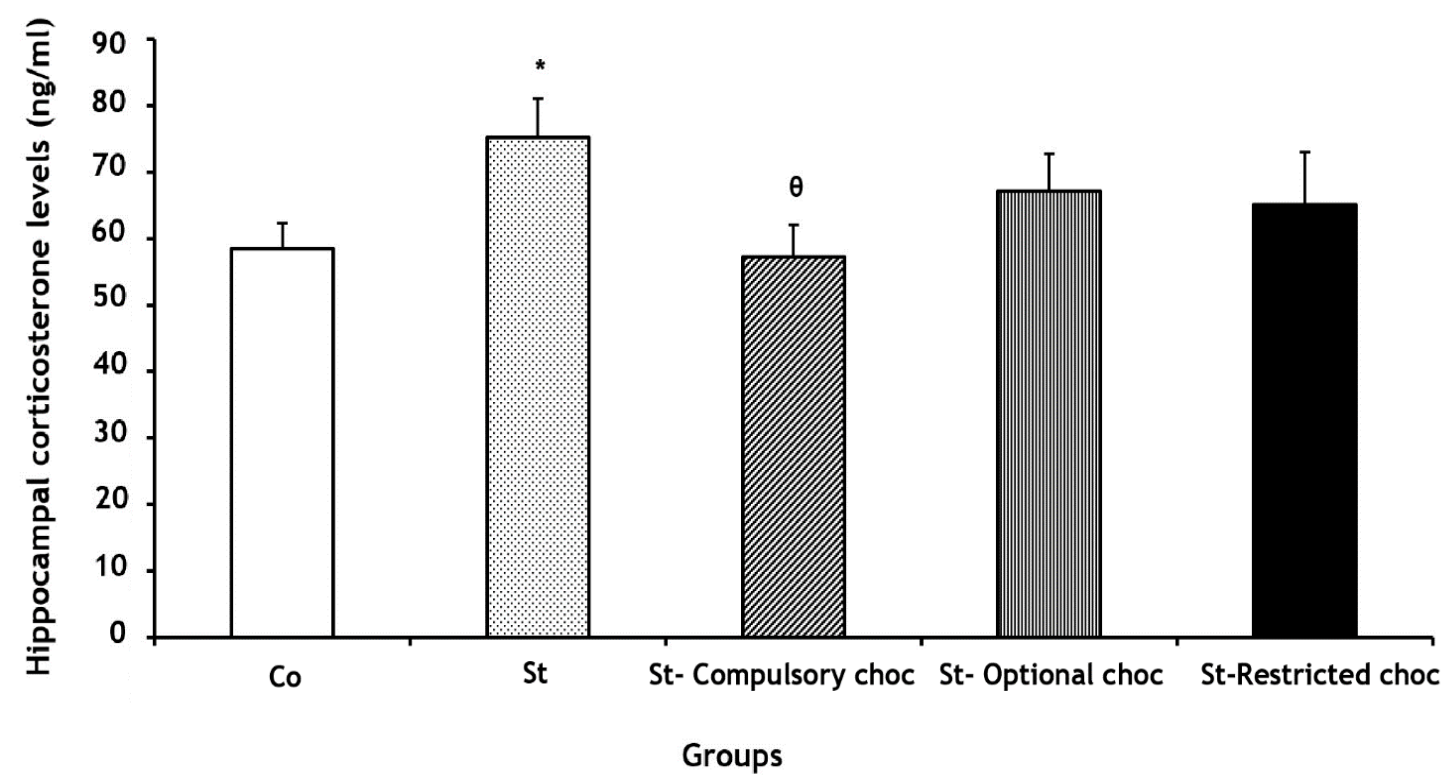

Fig.7. Effects of different dark chocolate diets on hippocampal corticosterone levels (ng/ml) in different groups under isolation stress $(n=7)$. Results are expressed as mean \pm SEM. ${ }^{\star} P<0.05$ compared to control group, ${ }^{\ominus} P<0.05$ compared to stress group. Co: control group, St: stress group, St- Compulsory Choc: stress with compulsory dark chocolate diet group, St-Optional Choc: stress with optional dark chocolate diet group, St-Restricted Choc: stress with restricted dark chocolate diet group.

corticosterone levels $[F(4,35)=1.882, \quad P>0.05$; Fig. 8].

As depicted in Figure 6, the serum corticosterone levels represented significant $(P<0.05)$ enhancements in the St group in comparison with the control group. Moreover, the results of serum corticosterone levels showed some decreases in all three dark chocolate dietary patterns, but the serum corticosterone levels decreased significantly $(P<0.01)$ only in the St-Compulsory Choc group compared to the St group. Furthermore, the serum corticosterone levels increased significantly in the St-Optional and St- 


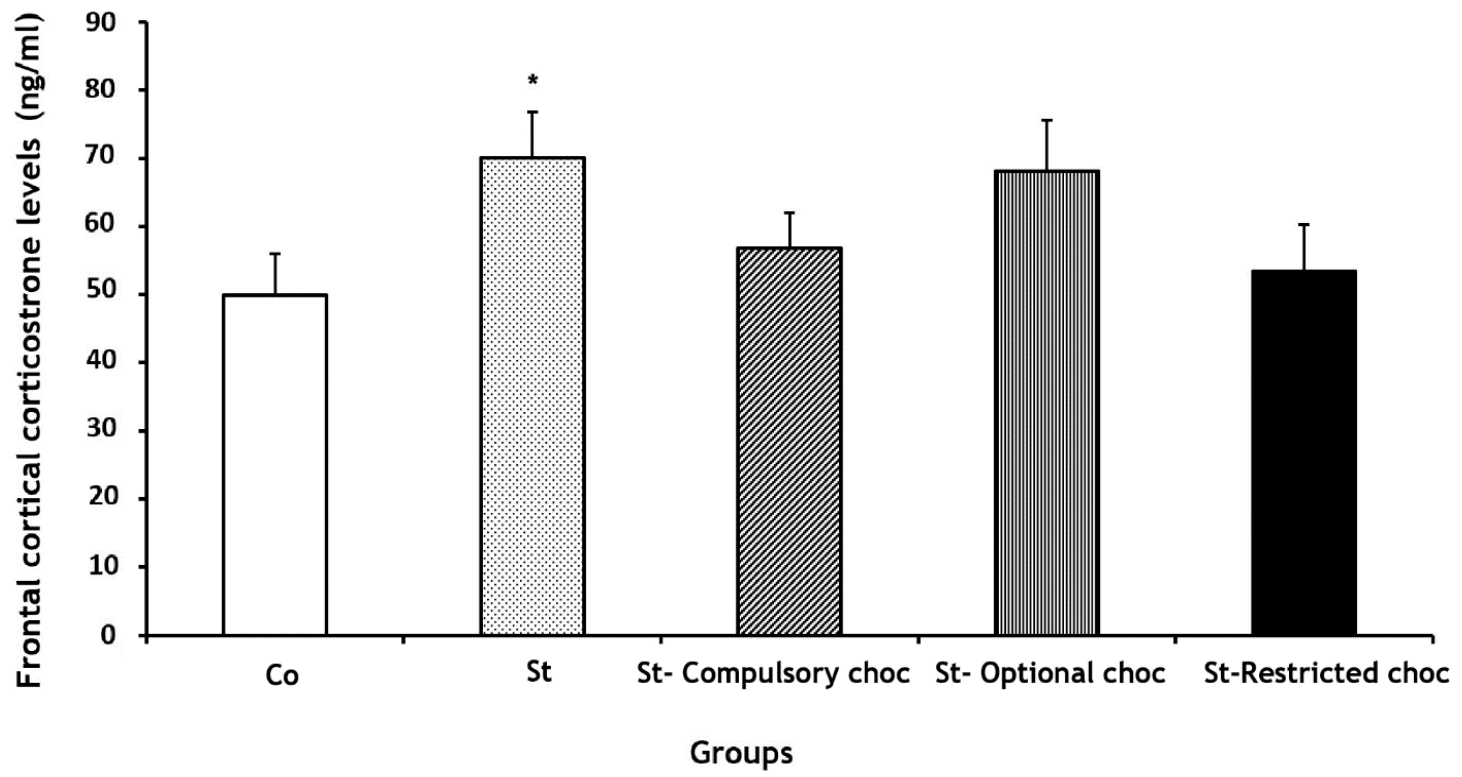

Fig.8. Effects of different dark chocolate diets on frontal cortical corticosterone levels $(\mathrm{ng} / \mathrm{ml})$ in different groups under isolation stress $(n=7)$. Results are expressed as mean \pm SEM. ${ }^{*} P<0.05$ compared to control group. Co: control group, St: stress group, St- Compulsory Choc: stress with compulsory dark chocolate diet group, St-Optional Choc: stress with optional dark chocolate diet group, St-Restricted Choc: stress with restricted dark chocolate diet group.

Restricted Choc diet groups (respectively, $P<0.01$ and $P<0.05)$ in comparison with the St-Compulsory Choc group (Fig. 6).

The hippocampal and frontal cortical corticosterone levels showed significant enhancements $(P<0.05$, in both them) in the St group compared to the control group. The hippocampal corticosterone levels decreased significantly $(P<0.05)$ only in StCompulsory Choc group in comparison with that in the St group (Figs. 7 and 8). The frontal cortical corticosterone levels did not show any significant difference in a similar comparison (Figs. 7 and 8), indicating that under stress conditions, there is a higher sensitivity in the hippocampus on corticosterone levels compared to the frontal cortical corticosterone levels.

\section{Discussion}

The effects of different (compulsory, optional and restricted) dark chocolate diets were investigated on brain functions such as learning, memory, memory consolidation and locomotor activity as well as the serum, hippocampal and frontal cortical corticosterone levels in rats under chronic isolation stress to determine which of the dark chocolate diet patterns may affect the brain function improvement under stress condition.

Regarding the current findings, there was occurrence of learning in all experimental groups at different levels, even in the chronically stressed group a low level of learning was seen. Accordingly, some studies have confirmed that even though stress accelerates the onset and severity of cognitive impairments such as learning (Ayoub, 2009; Gulpinar and Yegen, 2004; Jeong et al., 2006), learning was still formed under stress conditions (Dastgerdi et al., 2018; Joëls et al., 2006). Memory impairment was observed in chronic isolation stress as well. In some investigations, the simultaneous occurrence of memory deficits and chronic stress was seen, especially when the stress was uncontrollable, repeated and prolonged (Corcoba et al., 2017; Dastgerdi et al., 2017). In contrast to current findings, McEwen et al. (2001) reported a state of chronic stress habituation for the animals (McEwen, 2001) in which the observed differences may depend on the types of stress and memory tasks (Dastgerdi et al., 2018). In the current study, corticosterone levels increased in the serum, hippocampus and frontal cortex of the stressed group. Furthermore, chronic stress may alter the learning and memory by stress hormones (such as corticosterone) and other neurochemical factors (Corcoba et al., 2017; Seo et al., 2016).

According to other present findings, dark chocolate dietary patterns affected different aspects of brain functions under chronic stress. The compulsory and even restricted dark chocolate diets improved memory deficits induced by chronic stress. Whereas, 
the optional dark chocolate diet did not have any significant effects on memory improvement. The types of diet seem to play an important role in some brain functions such as memory (Mattson, 2000). Additionally, previous studies have demonstrated that cocoa-derived products and dark chocolate consumption may probably improve stress-related memory deficits (Swinton et al., 2018) and cognitive functions such as the visuospatial working memory in humans (Crichton et al., 2016; Ramirez-Sanchez et al., 2017). Furthermore, moderate and high doses of flavonoids and/or a dose of chocolate displayed memory improvement (Field et al., 2011; Scholey et al., 2010; Vauzour, 2014). Whereas, another study has emphasized the negative impact of cocoa on cognitive performance (Pase et al., 2013). These could vary depending on the chocolate's antioxidant status (flavonols) and bioavailability, the varieties of cocoa beans, participants' conditions (healthy or clinical), production stages, research type (Allgrove and Davison, 2014; Crichton et al., 2016) and even based on the current study, the dietary patterns is effective on brain functions. Overall, the aforementioned factors might affect the cocoa consumption efficacy in studies related to humans and animals (Allgrove and Davison, 2014; Crichton et al., 2016).

In this study, other findings indicate an improvement in memory consolidation and locomotor activity by compulsory dark chocolate diet under isolation stress conditions. In a previous study dark chocolate and its components are reported to consolidate memory (Fernell et al., 2016) and to improve neurocognitive status (Crichton et al., 2016). Nevertheless, the sustained consumption of cocoa-based food, enriched with dark chocolate, promoted locomotor activity and improved motor coordination (Cicvaric et al., 2018). Even though caffeine withdrawal decreased locomotor activity (Strain and Griffiths, 1995), Yamada et al. (2009) demonstrated that cocoa mass administration did not affect the locomotor activity in the open field test (Yamada et al., 2009). Such paradoxical results with dark chocolate diets may relate to factors including the stress type and its duration, type of behavioral tasks, rat's age and gender, and probably the variety of cocoa and/or chocolate and duration of their consumption (Engler et al., 2004; Ranjbar et al., 2016).

Notably, the compulsory dark chocolate diet could improve brain functions such as memory, memory consolidation and locomotor activity; but the brain responses seem to act differently in various dark chocolate diets. For instance, the compulsory dark chocolate diet appears to be the most effective type of dark chocolate diets under stress conditions. The authors, however, did not find any evidence about the memory processing in dark chocolate dietary patterns under stress. Some previous studies confirmed that cocoa and cocoa-derived products such as dark chocolate are the richest sources of different antioxidant compounds such as flavonoids (Small et al., 2001; Sokolov et al., 2013). Therefore, the compulsory dark chocolate diet may have had more flavonoids compared to other types of dark chocolate diets that could have acted more effectively on brain functions. However, the underlying effects of dark chocolate diet mechanism(s) have remained unexplored.

All in all, the current findings about hormonal changes represent the important role of the compulsory dark chocolate diet. Results in current study demonstrated that under stress conditions, compulsory dark chocolate diet reduced the corticosterone levels only in the serum and hippocampus, except in the frontal cortex. In addition, in previous studies, the decrease of stress hormones due to the consumption of dark chocolate were reported as well, albeit without considering the diet type (Almoosawi et al., 2012). Additionally, another study on the effects of dark chocolate indicated the reduced salivary cortisol levels in adults recruited from health-care and social settings (Tsang et al., 2019). In the compulsory dark chocolate diet, the changes of corticosterone levels in the hippocampus in comparison with the frontal cortex proposed a probable higher sensitivity in the hippocampus against dietary patterns of dark chocolate and its flavonoids. As such, Fowler et al. (2014) reported a differential sensitivity in hippocampus and prefrontal cortex towards the toxicity caused by alcohol.

\section{Conclusion}

To sum up, the different types of dark chocolate diets had various effects on brain functions under stress conditions. The restricted dark chocolate diet and particularly the compulsory one positively improved memory under stress conditions. Whereas, the memory consolidation, locomotor activity, serum and 
hippocampal corticosterone levels except the frontal cortical corticosterone level, improved only in compulsory dark chocolate diet. It seems that the compulsory and optional dark chocolate diets, respectively, had the best and least effects on the improvement of brain functions. Finally, concerning the changes in corticosterone levels due to different dark chocolate dietary patterns under stress conditions, it seems that, compared to the frontal cortex, the hippocampus shows more sensitivity to these diets. However, further cellular, structural and biochemical studies are needed to clarify the underlying physiological mechanism in different dark chocolate diets.

\section{Acknowledgments}

This work was supported by grants from Isfahan University of Medical Sciences, Isfahan, Iran. Conduction of the present research was made possible through the supports received from Isfahan University of Medical Sciences, Isfahan, Iran.

\section{Conflict of interest}

The authors declare that they have no conflict of interest.

\section{References}

Allan AM, Goggin SL, Caldwell KK. Prenatal alcohol exposure modifies glucocorticoid receptor subcellular distribution in the medial prefrontal cortex and impairs frontal cortex-dependent learning. PLos One 2014; 9. https://doi.org/10.1371/journal.pone.0096200

Allgrove J, Davison G. Dark chocolate/cocoa polyphenols and oxidative stress. In: Polyphenols in human health and disease 2014, pp. 241-251. https://doi.org/10.1016/B978-0-12-398456-2.00019-0

Almoosawi S, Tsang C, Ostertag LM, Fyfe L, AI-Dujaili EA. Differential effect of polyphenol-rich dark chocolate on biomarkers of glucose metabolism and cardiovascular risk factors in healthy, overweight and obese subjects: a randomized clinical trial. Food Funct 2012; 3: 1035-43. https://doi.org/10.1039/c2fo30060e

Ayoub RS. Effect of exercise on spatial learning and memory in male diabetic rats. Int $\mathrm{J}$ Diabetes Metab 2009; 17: 93-8. https://doi.org/10.1159/000497679

Bandegi AR, Rashidy-Pour A, Vafaei AA, Ghadrdoost B. Protective effects of Crocus sativus $L$. extract and crocin against chronic-stress induced oxidative damage of brain, liver and kidneys in rats. Adv Pharm Bull 2014; 4: 493-9. https://doi.org/10.5681/apb.2014.073

Barrientos RM, Thompson VM, Kitt MM, Amat J, Hale MW, Frank MG, et al. Greater glucocorticoid receptor activation in hippocampus of aged rats sensitizes microglia. Neurobiol Dis 2015; 36: 1483-95. https://doi.org/10.1016/j.neurobiolaging.2014.12.003

Cerqueira JJ, Pêgo JM, Taipa R, Bessa JM, Almeida OF, Sousa N. Morphological correlates of corticosteroidinduced changes in prefrontal cortex-dependent behaviors. J Neurosci 2005; 25: 7792-800. https://doi.org/10.1523/jneurosci.1598-05.2005

Cicvaric A, Bulat T, Bormann D, Yang J, Auer B, Milenkovic $\mathrm{I}$, et al. Sustained consumption of cocoa-based dark chocolate enhances seizure-like events in the mouse hippocampus. Food Funct 2018; 9: 1532-44. https://doi.org/10.1039/c7fo01668a

Corcoba A, Gruetter R, Do KQ, Duarte JM. Social isolation stress and chronic glutathione deficiency have a common effect on the glutamine-to-glutamate ratio and myo-inositol concentration in the mouse frontal cortex. J Neurochem 2017; 142: 767-75. https://doi.org/10.1111/ jnc. 14116

Crichton GE, Elias MF, Alkerwi AA. Chocolate intake is associated with better cognitive function: The MaineSyracuse Longitudinal Study. Appetite 2016; 100: 12632. https://doi.org/10.1016/j.appet.2016.02.010

Dailey MJ, Stingl KC, Moran TH. Disassociation between preprandial gut peptide release and food-anticipatory activity. Endocrinology 2012; 153: 132-42. https://doi.org/10.1210/en.2011-1464

Dastgerdi AH, Radahmadi M, Pourshanazari AA, Dastgerdi $\mathrm{HH}$. Effects of crocin on learning and memory in rats under chronic restraint stress with special focus on the hippocampal and frontal cortex corticosterone levels. Adv Biomed Res 2017; 6: 157-63. https://doi.org/ 10.4103/abr.abr_107_17

Dastgerdi HH, Radahmadi M, Reisi P, Dastgerdi AH. Effect of crocin, exercise, and crocin-accompanied exercise on learning and memory in rats under chronic unpredictable stress. Adv Biomed Res 2018; 7: 137-47. https://doi.org/10.4103/abr.abr_153_18

Dedovic K, Duchesne A, Andrews J, Engert V, Pruessner JC. The brain and the stress axis: the neural correlates of cortisol regulation in response to stress. Neuroimage 2009; 47: 864-71. https://doi.org/10.1016/j.neuroimage. 2009.05.074

Divsalar K. Destructive effects of prenatal WIN 55212-2 exposure on central nervous system of neonatal rats. Addict Health 2012; 4: 9-19.

Engler MB, Engler MM, Chen CY, Malloy MJ, Browne A, Chiu EY, et al. Flavonoid-rich dark chocolate improves endothelial function and increases plasma epicatechin concentrations in healthy adults. J Am Coll Nutr 2004; 23: 197-204. https://doi.org/10.1080/07315724.2004. 10719361

Fernell M, Swinton C, Lukowiak K. Epicatechin, a component of dark chocolate, enhances memory formation if applied during the memory consolidation period. Commun Integr Biol 2016; 9: 816-23. https://doi.org/10.1080/19420889.2016.1205772

Field DT, Williams CM, Butler LT. Consumption of cocoa flavanols results in an acute improvement in visual and cognitive functions. Physiol Behav 2011; 103: 255-60. 
https://doi.org/10.1016/j.physbeh.2011.02.013

Fowler AK, Thompson J, Chen L, Dagda M, Dertien J, Dossou KS, et al. Differential sensitivity of prefrontal cortex and hippocampus to alcohol-induced toxicity. PLos One 2014; 9. https://doi.org/10.1371/journal.pone. 0106945

Franco $R$, Oñatibia-Astibia A, Martínez-Pinilla E. Health benefits of methylxanthines in cacao and chocolate. Nutrients 2013; 5: 4159-73. https://doi.org/10.3390/ nu5104159

Gulpinar MA, Yegen BC. The physiology of learning and memory: role of peptides and stress. Curr Protein Pept Sc 2004; 5: 457-73. https://doi.org/10.2174/13892030 43379341

Holtzer R, Scheon C, Demetriou E, Mahoney JR, Izzetoglu $M$, Wang $C$, et al. Stress and gender effects on prefrontal cortex oxygenation levels assessed during single and dual-task walking conditions. Eur J Neurosci 2017; 45: 660-70. https://doi.org/10.1111/ejn.13518

Huang AC, Shyu BC, Hsiao S, Chen TC, He AB. Neural substrates of fear conditioning, extinction, and spontaneous recovery in passive avoidance learning: a c-fos study in rats. Behav Brain Res 2013; 237: 23-31. https://doi.org/10.1016/j.bbr.2012.09.024

Iglesias T, Montero S, Otero MJ, Parra L, Fuentes JA. Preproenkephalin RNA increases in the hypothalamus of rats stressed by social deprivation. Cell Mol Neurobiol 1992; 12: 547-55. https://doi.org/10.1007/ bf00711234

Jeong YH, Park CH, Yoo J, Shin KY, Ahn SM, Kim HS, et al. Chronic stress accelerates learning and memory impairments and increases amyloid deposition in APPV717I-CT100 transgenic mice, an Alzheimer's disease model. Faseb $J$ 2006; 20: 729-31. https://doi.org/10.1096/fj.05-4265fje

Joëls M, Pu Z, Wiegert O, Oitzl MS, Krugers HJ. Learning under stress: how does it work? Trends Cogn Sci 2006; 10: 152-8. https://doi.org/10.1016/j.tics.2006.02.002

Kemsawasd V, Chaikham P, Rattanasena P. Survival of immobilized probiotics in chocolate during storage and with an in vitro gastrointestinal model. Food biosci 2016; 16: 37-43. https://doi.org/10.1016/j.fbio.2016.09.001

Kim TK, Han PL. Functional connectivity of basolateral amygdala neurons carrying orexin receptors and melanin-concentrating hormone receptors in regulating sociability and mood-related behaviors. Exp Neurobiol 2016; 25: 307-17. https://doi.org/10.5607/en.2016. 25.6.307

Li N, Liu RJ, Dwyer JM, Banasr M, Lee B, Son H, et al. Glutamate N-methyl-D-aspartate receptor antagonists rapidly reverse behavioral and synaptic deficits caused by chronic stress exposure. Biol pychiatry 2011; 69: 754-61. https://doi.org/10.1016/j.biopsych.2010.12.015

Martin FP , Rezzi S, Peré-Trepat E, Kamlage B, Collino S, Leibold $E$, et al. Metabolic effects of dark chocolate consumption on energy, gut microbiota, and stressrelated metabolism in free-living subjects. J Proteome Res 2009; 8: 5568-79. https://doi.org/10.1021/ pr900607v
Mattson MP. Neuroprotective signaling and the aging brain: take away my food and let me run. Brain Res 2000; 886: 47-53. https://doi.org/10.1016/S0006-8993(00) 02790-6

McEwen BS. Plasticity of the hippocampus: adaptation to chronic stress and allostatic load. Ann Ny Acad Sci 2001; 933: 265-77. https://doi.org/10.1111/j.17496632.2001.tb05830.x

Mills D, Ward R. Attenuation of psychosocial stress-induced hypertension by gamma-linolenic acid (GLA) administration in rats. Proc Soc Exp Biol Med 1984; 176: 32-7. https://doi.org/10.3181/00379727-176-41838

Miranda AM, Oliveira TG. Lipids under stress-a lipidomic approach for the study of mood disorders. Bioessays 2015; 37: 1226-35. https://doi.org/10.1002/bies. 201500070

Pase MP, Scholey AB, Pipingas A, Kras M, Nolidin K, Gibbs $A$, et al. Cocoa polyphenols enhance positive mood states but not cognitive performance: a randomized, placebo-controlled trial. J Psychopharmacol 2013; 27: 451-8. https://doi.org/ $10.1177 / 0269881112473791$

Radahmadi M, Alaei H, Sharifi MR, Hosseini N. Effects of different timing of stress on corticosterone, BDNF and memory in male rats. Physiol Behav 2015; 139: 459-67. https://doi.org/10.1016/j.physbeh.2014.12.004

Radahmadi M, Hosseini N, Nasimi A. Effect of chronic stress on short and long-term plasticity in dentate gyrus; Study of recovery and adaptation. Neuroscience 2014; 280: 121-9. https://doi.org/10.1016/j.neuroscience. 2014.09.005

Ramirez-Sanchez I, Rodriguez A, Neri T, Mendoza-Lorenzo P, Ceballos G, Villarreal F. (-)-Epicatechin mitigates oxidative stress, neuroinflammation and hyperphosphorylation of TAU in hippocampus of senile mice. FASEB J 2017; 31: 814.7. https://doi.org/ 10.1096/fasebj.31.1_supplement.814.7

Ranjbar H, Radahmadi M, Alaei H, Reisi P, Karimi S. The effect of basolateral amygdala nucleus lesion on memory under acute, mid and chronic stress in male rats. Turk J Med Sci 2016; 46: 1915-25. https://doi.org/10.3906/sag-1507-7

Scholey AB, French SJ, Morris PJ, Kennedy DO, Milne AL, Haskell CF. Consumption of cocoa flavanols results in acute improvements in mood and cognitive performance during sustained mental effort. J Psychopharmacol 2010; 24: 1505-14. https://doi.org/ 10.1177/0269881109106923

Seo H, Park CH, Choi S, Kim W, Jeon BD, Ryu S. Effects of voluntary exercise on apoptosis and cortisol after chronic restraint stress in mice. $J$ Exerc Nutrition Biochem 2016; 20: 16. https://doi.org/10.20463/ jenb.2016.09.20.3.3

Shah SR, Alweis R, Najim NI, Dharani AM, Jangda MA, Shahid $M$, et al. Use of dark chocolate for diabetic patients: a review of the literature and current evidence. J Community Hosp Intern Med Perspect 2017; 7: 21821. https://doi.org/10.1080/20009666.2017.1361293

Shin JW, Seol IC, Son CG. Interpretation of animal dose 
and human equivalent dose for drug development. $\mathrm{J}$ Korean Med Sci 2010; 31: 1-7.

Simadibrata M. Anti-free radical effects of dark chocolate in radical damage and constipation. Acta Med Indonesiana 2011; 43:217.

Small DM, Zatorre RJ, Dagher A, Evans AC, JonesGotman M. Changes in brain activity related to eating chocolate: from pleasure to aversion. Brain 2001; 124: 1720-33. https://doi.org/10.1093/brain/124.9.1720

Sokolov AN, Pavlova MA, Klosterhalfen S, Enck P. Chocolate and the brain: neurobiological impact of cocoa flavanols on cognition and behavior. Neurosci Biobehav Rev 2013; 37: 2445-53. https://doi.org/ 10.1016/j.neubiorev.2013.06.013

Steinberg FM, Bearden MM, Keen CL. Cocoa and chocolate flavonoids: implications for cardiovascular health. J Am Diet Assoc 2003; 103: 215-23. https://doi.org/10.1053/jada.2003.50028

Strain EC, Griffiths RR. Caffeine dependence: fact or fiction? J Roy Soc Med 1995; 88: 437.

Sugimoto T, Yoshida M, Ono R, Murata S, Saji N, Niida S, et al. Frontal lobe function correlates with one-year Incidence of urinary incontinence in elderly with
Alzheimer disease. J Alzheimers Dis 2017; 56: 567-74. https://doi.org/10.3233/JAD-160923

Swinton E, de Freitas E, Swinton C, Shymansky T, Hiles E, Zhang J, et al. Green tea and cocoa enhance cognition in Lymnaea. Commun Integr Biol 2018; 11: e1434390. https://doi.org/10.1080/19420889.2018.1434390

Tsang C, Hodgson L, Bussu A, Farhat G, Al-Dujaili E. Effect of polyphenol-rich dark chocolate on salivary cortisol and mood in adults. Antioxidants 2019; 8: 149. https://doi.org/10.3390/antiox8060149

Vauzour D. Effect of flavonoids on learning, memory and neurocognitive performance: relevance and potential implications for Alzheimer's disease pathophysiology. J Sci Food Agric 2014; 94: 1042-56. https://doi.org/ 10.1002/jsfa.6473

Vohora DI, Pal SN, Pillai KK. Effect of locomotor activity on the passive avoidance test for the evaluation of cognitive function. Indian J Pharmacol 2000; 32: 242-5.

Yamada T, Yamada Y, Okano Y, Terashima T, Yokogoshi $\mathrm{H}$. Anxiolytic effects of short- and long-term administration of cacao mass on rat elevated T-maze test. J Nutr Biochem 2009; 20: 948-55. https://doi.org/ 10.1016/j.jnutbio.2008.08.007 